Revista de Matemática: Teoría y Aplicaciones 2003 10(1-2) : 131-146

CIMPA - UCR - CCSS ISSN: 1409-2433

\title{
HERRAMIENTAS MATEMÁTICAS PARA LA VALORACIÓN DE LA AMPLIACIÓN DE UNA INFRAESTRUCTURA PORTUARIA*
}

\author{
TRINIDAd CASAsús ${ }^{\dagger}$ \\ ENRIC CRespo \\ C. JUAN \\ F. Olmos \\ J.C. PÉREZ
}

Recibido: 13 Dec 2002

\begin{abstract}
Resumen
El problema abordado consiste en la valoración de la ampliación de una infraestructura portuaria ya consolidada, que conlleva unas inversiones a largo plazo.

Para ello hay que recurrir a medios de análisis capaces de recoger, en la medida de lo posible, la incertidumbre sobre la futura evolución de los tráficos de mercancías, sobre el efecto de la competencia entre puertos, etc., y que los métodos tradicionales no aproximan en toda su dimensión. Existe, además, un problema de decisión de política óptima de gestión del proyecto que depende de variables de decisión que modelizan las opciones presentes en el mismo.

Las oportunidades de inversión han sido tratadas como una colección de opciones americanas sobre activos reales. Nosotros hemos optado por una variación del método de Monte Carlo para la obtención del Valor Actual Neto estocástico. Para la toma de decisiones se plantea un problema de control óptimo.

El procedimiento genera unos escenarios que incrementan la información sobre el tráfico y les asocia un VAN esperado así como la probabilidad de que éste sea positivo o negativo. Además de esta distribución de probabilidad proporciona la de los flujos de caja (posible indicador del riesgo) y del VAN asociado.

Los cálculos asociados a cada escenario son muy costosos y resulta imposible examinar todas las posibilidades de porcentajes de participación del capital privado y de

${ }^{*}$ Este trabajo ha sido parcialmente financiado por la Autoridad Portuaria de Valencia y el Instituto Valenciano de Investigaciones Económicas. Queremos dar las gracias al Instituto de Economía Internacional y especialmente a su Director Leandro García por la ayuda prestada, así como a nuestros compañeros Begoña Font, Vicent Liern y Carlos Ivorra.

${ }^{\dagger}$ Departamento de Matemática Económico-Empresarial. Universitat de València, 46071 Valencia, Spain. E-Mail: Trinidad.Casasus@uv.es.

${ }^{\ddagger}$ Misma dirección que T. Casasús. E-Mail: Enric.Crespo@uv.es.
\end{abstract}


fecha óptima para cada inversión, o su desestimación. Proponemos un método basado en el Scatter Search para tomar estas decisiones.

Palabras clave: Ecuaciones estocásticas, métodos de Monte Carlo, métodos metaheurísticos.

Keywords: Stochastic equations, Monte Carlo simulation, metaheuristics.

Mathematics Subject Classification: 90C57, 90C27, 91B74.

\section{Introducción}

El problema presentado es la valoración de un proyecto de ampliación de una infraestructura portuaria.

Este proyecto conlleva una inversión a largo plazo que, dados los problemas económicos que presenta (la evolución del volumen de los tráficos de mercancías, el efecto de la competencia entre puertos, el desarrollo de las economías regionales en el ámbito de influencia del puerto estudiado, el momento óptimo de realizar la nueva inversión, la elección de la forma de financiación -pública, privada o mixta- y/o de explotación, etc.) precisa, si se quiere realizar una valoración ajustada del proyecto, recurrir a instrumentos de análisis capaces de recoger y sistematizar, en la medida de lo posible, la incertidumbre sobre el futuro, las diferentes opciones presentes en el proyecto y el control óptimo requerido en la toma de decisiones.

La incertidumbre sobre el futuro ha sido abordada en nuestro estudio con una variación novedosa del método de simulación de Monte Carlo para la fase de valoración (generación de flujos de caja de la inversión).

La opcionalidad del proyecto requiere un estudio de las opciones sobre activos reales ([DP94],[T96], [CA01] entre otros). En el caso de una oportunidad de inversión secuencial, la opción puede ejercerse (invertir) o no según la nueva información incorporada. Este proceso, en general, reduce el Valor Actual Neto (VAN) clásico de un proyecto, pero puede provocar un aumento del valor de la flexibilidad de la dirección que contrarreste el efecto negativo anterior. La teoría de las Opciones Reales se basa en el método de Valoración de Opciones Financieras de Black, Merton y Scholes [BSM73] que presenta un modo de determinación del precio de los contratos de opciones sobre activos financieros, pero debe tenerse en cuenta que, por las características de un puerto, la valoración por el argumento de no arbitraje (método de valoración de opciones financieras de BSM) no es posible dada la imposibilidad de replicar el proceso de flujos de caja de un puerto mediante una cartera de activos negociados cuyo proceso de precios esté estrechamente correlacionado.

El problema general de decisión múltiple se aborda como un problema de optimización discreta que combine las fechas de construcción de cada muelle con sus porcentajes de inversión pública-privada.

Aunque el método propuesto ha sido motivado por el problema específico de las inversiones portuarias, es claro que nos encontramos en presencia de una problemática generalizable a cualquier contexto financiero de la empresa, y que requiera una sistematización de ambas situaciones:la valoración en presencia de incertidumbre y la toma de decisiones frente a la opcionalidad, así como las interconexiones entre ellas. 


\section{Descripción del problema real. Peculiaridades de un puer- to}

En el caso de un puerto, existen algunas características que conviene subrayar y tener en cuenta a la hora de analizar un proyecto de inversión:

- Un puerto debe combinar la oferta de un servicio público con la eficiencia de una empresa privada

- Existe un mercado cautivo debido a la proximidad geográfica del puerto.

- La cercanía de un puerto genera en su zona de influencia oportunidades de crecimiento regional.

- El tipo especial de competencia entre puertos próximos o de la misma área geográfica, y su influencia sobre el desarrollo o "decremento" potencial de la economía en las zonas de influencia.

- El poder de atracción sobre otras economías que la existencia de un puerto conlleva.

En nuestro caso partimos de una infraestructura portuaria ya existente, en funcionamiento y consolidada, con clientes "cautivos" dadas las especiales características de la economía regional, y con otros clientes que varían en función de la competitividad del puerto respecto de medios alternativos de distribución de mercancías, o de la oferta de otros puertos. Disponemos de tres estructuras (muelles) en funcionamiento, cada una de las cuales con una capacidad máxima asignada de gestión de tráfico de mercancías.

En lo que respecta a los distintos tráficos que pasan por el puerto actual han sido agrupados siguiendo criterios de racionalidad y coherencia expresados por los gestores del puerto, pero también la viabilidad estadística de dichas agrupaciones, pues las series históricas de las mercancías y sus expectativas futuras tienen discrepancias entre ellas. Se ha estudiado también el proceso de aplicación de tarifas, para que la utilización de una tarifa media para que el tráfico agregado de mercancías no suponga una distorsión de la situación real. El resultado de todo esto nos llevó al agrupamiento de los tráficos actuales en 21 tipos de tráfico.

El proyecto de ampliación propuesto consiste en la construcción de tres nuevos muelles, formando una dársena contigua a la existente, a los que se destinará el excedente del tráfico, una vez alcanzada la capacidad teórica asignada al muelle que lo gestiona.

Por último para completar el modelo estudiamos la opcionalidad existente a la hora de llevar a cabo el proyecto, constatando la obligatoriedad de construcción del primer muelle (decisión ya tomada) que se realizará con $100 \%$ de capital público, siendo prevista su puesta en funcionamiento para el año 2005. En cuanto a los otros dos nuevos muelles, su construcción es opcional con una fecha de inicio de la actividad variable (entre 2007 y 2012) siendo uno de ellos de capital $100 \%$ público mientras que el segundo admite una inversión mixta pública-privada cuyo porcentaje óptimo debe determinarse.

El porcentaje de financiación público-privado de la inversión para el tercer muelle no afecta a la distribución de tráficos, sin embargo, la construcción de uno de los dos muelles 
opcionales sí que influye en la reubicación de excedentes y, por tanto, en la generación de flujos de caja. Así pues concluimos que, finalizado el diseño del modelo, la información que debe proporcionar una vez resuelto es la siguiente:

Fecha óptima de inversión para cada uno de los muelles opcionales, o bien si es preferible no construir alguno de ellos.

Valor final del proyecto de inversión una vez establecida la secuencia óptima de construcción, con todas las implicaciones que conlleva desde el punto de vista de la generación de flujos.

\section{Metodología propuesta para un problema de decisión múltiple}

\section{1}

La modelización de la evolución futura del tráfico de mercancías requiere una herramienta matemática que nos permita obtener información sobre los niveles de tráfico que se alcanzarán en un instante futuro que incorpore las previsiones de los expertos del sector y la aleatoriedad reflejo de la incertidumbre inherente.

Los procesos de difusión constituyen el instrumento más utilizado en este tipo de modelizaciones. Una variable $N_{i}$ sigue un proceso de difusión si su evolución varía según la ecuación

$$
d N_{i}(t)=\mu_{i}\left(N_{i}, t\right) d t+\sigma_{i}\left(N_{i}, t\right) d w_{i} .
$$

En nuestro caso, el estudio de las series históricas sobre el tráfico portuario nos llevaron a la conclusión de que los tráficos se deben modelizar por un proceso de difusión de reversión a la media, que expresado en tiempo discreto viene dado por

$$
N_{i}(t)=N_{i}(t-1)+\mu_{i}\left(N_{i}, t\right) \Delta t+\sigma_{i}(N i, t) \Delta z
$$

siendo

- $\mu_{i}\left(N_{i}, t\right)=a\left(b-N_{i}(t-1)\right)$ el término determinista que describe la tendencia de la variable que los expertos consideran adecuada y que en el caso de reversión a la media se comporta creciendo con una tasa $a$, hasta un nivel medio $b$, entorno al cual comienza a oscilar.

- $\sigma_{i}\left(N_{i}, t\right) \Delta z$, con $\Delta z=\epsilon(\Delta t)^{1 / 2}$ un proceso de Wiener, la parte estocástica que "perturba" la tendencia anterior de forma aleatoria, perturbación controlada por un factor corrector de la intensidad que es función del tiempo transcurrido. $\sigma_{i}\left(N_{i}, t\right)$ gradúa la intensidad de la perturbación, es una función de $N_{i}$ y de $t$, habitualmente se expresa como un valor o como porcentaje que multiplica a $N_{i}(t-1)$ y se le denomina volatilidad del proceso. El término $(\Delta t)^{1 / 2}$ hace que la perturbación sea proporcional a la raíz cuadrada del tiempo transcurrido, en lugar de $\Delta t$ que se utiliza en la tendencia. De este modo se suaviza su efecto.

Suponemos que en el modelo intervienen q tráficos, modelizados en su evolución en el tiempo mediante el proceso de difusión considerado, ajustando los parámetros $a_{i}$ y $b_{i}$ a las 
series históricas. Una vez ajustados los parámetros $a_{i}, b_{i}$ por medio de los procedimientos estadísticos adecuados, y asignados distintos valores a $\sigma_{i}$ a partir de los datos suministrados por la Autoridad Portuaria [AP99], una de las principales contribuciones de este trabajo consiste en la construcción de una serie de escenarios en el instante $I+n$ (instante de puesta en funcionamiento del nuevo muelle), que incrementan la información disponible sobre el comportamiento de los tráficos $N_{i}, i=1, \ldots, q$. La generación de los escenarios se realiza aplicando una modificación del método clásico de Monte Carlo:

- A partir de los datos iniciales de tráfico en el instante $I$, simulamos $H$ trayectorias aleatorias (según el ajuste realizado) para cada uno de los $q$ tráficos, desde el instante $I$ hasta el instante $I+n$, y agrupamos los $H$ valores correspondientes al instante $I+n, N_{i}^{j}(I+n), j=1,2, \ldots, H$, en un histograma de frecuencias con un número $r$ de cortes adecuado al problema.

- Obtenemos, para cada tráfico, los $r$ valores puntos medios de los intervalos del histograma, que denotamos $\bar{N}_{i}^{1}, \bar{N}_{i}^{2}, \ldots, \bar{N}_{i}^{r}, i=1,2, \ldots, q$, y les asociamos la probabilidad de ocurrencia dada por la frecuencia relativa de las simulaciones en el corte correspondiente.

- Definimos un escenario en el instante $I+n$ del tiempo como un vector de $q$ componentes, siendo cada una de ellas uno de los $r$ representantes de cada uno de los $q$ histogramas. Matemáticamente se calcula el producto cartesiano de los $q$ espacios

$$
\left\{\bar{N}_{i}^{1}, \bar{N}_{i}^{2}, \ldots, \bar{N}_{i}^{r}\right\}_{j=1,2, \ldots, H}
$$

- Los $r_{q}$ elementos de este producto cartesiano constituyen el espacio de escenarios en el instante $I+n$.

- Dado que cada componente de un escenario lleva asociada una probabilidad, cada escenario posee la probabilidad producto de las probabilidades asociadas a cada una de sus $q$ componentes. Obtenemos de este modo una distribución de probabilidad del espacio de escenarios en el instante $I+n$.

Esta novedad propuesta nos permite considerar, en el instante de puesta en marcha del nuevo muelle (instante $I+n$ ), una gran cantidad de posibles puntos de partida (cada uno de los escenarios) para la generación de futuras trayectorias de los subyacentes, cada uno de ellos con una probabilidad de ocurrencia asociada cuya importancia radica en el hecho de poder calificar, cada escenario, como favorable o desfavorable a partir de los valores tomados por las variables implicadas en los criterios de decisión establecidos en el problema.

Si consideramos además una vida útil del muelle de $m$ unidades temporales (u.t.), para cada escenario considerado calculamos un VAN estocástico asociado de la forma siguiente:

- Obtenemos los flujos de caja netos esperados función de las variables de estado, para el período de los m u.t., utilizando de nuevo la simulación de Monte Carlo para la obtención de las nuevas trayectorias aleatorias de los tráficos, considerando ahora como punto de partida los escenarios. 
- Calculamos el VAN asociado correspondiente, dados los flujos del punto anterior y descontados al tipo libre de riesgo (que se considera constante durante toda la simulación, siguiendo las hipótesis con las que trabaja AP). La fórmula para el cálculo del flujo descontado es la discretización de

$$
F_{d e s}(I+n)=\int_{0}^{\infty} F\left(N_{1}, N_{2}, \ldots, N_{q}, \tau\right) e^{-\int_{0}^{\tau} r d u} d \tau
$$

donde hemos denotado por $F\left(N_{1}, N_{2}, \ldots, N_{q}, \tau\right)$ el flujo neto de caja correspondiente al instante de tiempo $\tau$. El cálculo del VAN descuenta la inversión y añade la desinversión correspondiente.

La suma de los valores del VAN obtenidos para cada escenario, ponderados con la probabilidad correspondiente a cada uno de ellos, permite calcular el valor esperado del muelle considerado y sus estadísticos.

Ya que los flujos de caja dependen de los tráficos, modelizados como procesos de difusión, entonces los flujos netos de caja acumulados durante el periodo de explotación y descontados a la fecha del escenario considerado, dependerán de la evolución en el tiempo de estos q tráficos. Así pues, fijado uno de los escenarios, $e s c_{i}$, si realizamos $N$ simulaciones de la evolución conjunta de sus $q$ componentes, obtendremos $N$ posibles valores de la función de flujos netos descontados y acumulados $F_{d e s}^{e s c_{i}}$. Estos $N$ valores pueden tratarse mediante un histograma de frecuencias que origina una distribución de probabilidad de $F_{d e s}^{e s c_{i}}$, asociada al escenario fijado.

En la Figura 1 se muestra dicha distribución para la función $F_{d e s}^{e s c_{0}}$ obtenida a partir del único escenario existente en $t=0$ que constituye el estado inicial, para una infraestructura dependiente de 6 tráficos, supuesto un período de explotación de 25 u.t.

De esta distribución de probabilidad de $F_{d e s}^{e s c_{i}}$ se puede obtener medidas de rentabilidad, como la del valor esperado $E\left(F_{d e s}^{e s c_{i}}\right)$ que nosotros utilizamos, y medidas de riesgo, como la desviación típica o la probabilidad de que $F_{d e s}^{e s c_{i}}$ sea negativa, que mediría el porcentaje de flujos netos negativos para el $e s c_{i}$.

Para cada uno de los $r^{q}$ escenarios $e s c_{i}$ en un instante $\tau$, dispondríamos de una distribución de probabilidad de $F_{\text {des }}^{e s c_{i}}$ obtenida del mismo modo que la del ejemplo. Calculando el valor esperado de cada una de ellas, $E\left(F_{d e s}^{e s c_{i}}\right), i=1, \ldots, r^{q}$, obtenemos la rentabilidad esperada para cada escenario. De este modo es inmediato determinar qué escenarios son favorables (aquellos tal que $E\left(F_{d e s}^{e s c_{i}}\right) \geq 0$ ), y cuáles desfavorables (en caso contrario).

Si a cada valor esperado $E\left(F_{d e s}^{e s c_{i}}\right), i=1,2, \ldots, r^{q}$, le asociamos la probabilidad de ocurrencia de su escenario de partida $e s c_{i}$, obtenemos una distribución de probabilidad $E\left(F_{d e s}^{e s c_{i}}\right)$ respecto de los diferentes escenarios utilizados. Una distribución de probabilidad de este tipo, obtenida en una de las simulaciones del ejemplo resuelto, se presenta en la Figura 2. El eje horizontal contiene los valores de $E\left(F_{d e s}^{e s c_{i}}\right), i=1,2, \ldots, r^{q}$, y el eje vertical la probabilidad de ocurrencia de los mismos. 
El valor esperado de la distribución de probabilidad anterior, es decir, $E\left(E\left(F_{d e s}^{e s c_{i}}\right)\right) / i=1,2, \ldots, r^{q}$, mide la rentabilidad esperada de una infraestructura que comienza a generar flujos de caja en el instante $\tau$ en el que están construidos los escenarios, y para la cual ya existe una decisión de construir. En este caso no es posible modificar dicha decisión aunque nos encontremos en presencia de escenarios desfavorables. En el apartado siguiente analizaremos el caso general en el que sí es posible demorar y/o abandonar la inversión y como ello afecta a la distribución y a su valor esperado.

Como medida de riesgo que complemente la rentabilidad obtenida del proyecto, puede utilizarse la del porcentaje de escenarios desfavorables, es decir, la probabilidad $P\left(E\left(F_{d e s}^{e s c_{i}}\right)\right)<0, i=1,2, \ldots, r^{q}$. Otros análisis de riesgo, nos llevan a la conclusión de que podría suceder que un escenario fuese favorable según el criterio expuesto anteriormente, es decir, que su valor $E\left(F_{d e s}^{e s c_{i}}\right)$ fuese positivo, y sin embargo, existiese un riesgo implícito asociado a dicho escenario derivado de las características específicas de la distribución de probabilidad de $F_{d e s}^{e s c_{i}}$ (desviación típica o probabilidad de realizaciones negativas de $F_{\text {des }}^{e s c_{i}}$ muy elevadas).

Para detectar estos posibles escenarios favorables arriesgados, resulta conveniente representarse las desviaciones típicas de las funciones $F_{d e s}^{e s c_{i}}$ de todos los escenarios utilizados, así como las correspondientes probabilidades de realizaciones negativas de $F_{d e s}^{e s c_{i}}$. De este modo se observa como se comporta el conjunto de los escenarios respecto de los estimadores de riesgo mencionados.

\section{2}

En cuanto al problema de establecimiento de fecha óptima de inversión para un proyecto de ampliación consistente en un solo muelle, suponiendo que se dispone de varias fechas de comienzo de la ampliación, entre las que hay que elegir la fecha óptima de inicio de obras, éste puede plantearse como un problema de elección entre proyectos de inversión 
alternativos, cada uno con una posible fecha de inversión y con una función de flujos netos descontados específica. Dicha función dependerá del tráfico, e incorporará una penalización en los ingresos por la demora en la construcción, dado que no disponer de instalaciones afectará previsiblemente la decisión de los usuarios habituales o potenciales de desviarse a algún otro puerto alternativo. De este modo existe, en fechas intermedias, la opción de diferir y, en la última fecha disponible, dos posibilidades: ejecutar el proyecto sin opcionalidad si existe obligación de construir (necesidad social de la obra pública), o bien, la opción de no ejecutar el proyecto (abandonar completamente la inversión) si dicha obligación no está presente.

Fijada una fecha intermedia de inicio y un escenario $e s c_{i}$, y una vez incorporadas a la función de flujos netos descontados $F_{d e s}^{e s c_{i}}$ las correspondientes penalizaciones por demora, calculamos $E\left(F_{d e s}^{e s c_{i}}\right)$ tal y como describimos en la sección anterior. Este valor puede ser negativo, lo cual significa que el escenario es desfavorable. Como existe la opción de demorar la inversión, puede modificarse la función $E\left(F_{d e s}^{e s c_{i}}\right)$ para incorporar dicha opción, eligiendo como función de pagos para ese escenario el máximo entre el valor hallado y el valor cero. Si el escenario es favorable, o sea, el valor $E\left(F_{d e s}^{e s c_{i}}\right)$ es positivo, se elegiría invertir, conservándose el valor esperado original. Este proceso da lugar a una nueva función asociada a cada fecha posible de inicio $\tau_{j}$ y evaluada en cada escenario de $\tau_{j}$, a la que denominaremos

$$
F_{\text {mod }}^{\tau_{j}}=\max \left\{E\left(F_{d e s}^{e s c_{i}}\right), 0\right\}
$$

La última fecha de inversión posible $\tau_{f}$, permite dos definiciones diferentes de la función $\left.F_{m o d}^{\tau_{f}}\right)$. Si no existe obligación de construir, el valor esperado en los escenarios desfavorables se sustituye por cero como en las fechas anteriores. Sin embargo, si existe obligación de construir debe conservarse el valor de $E\left(F_{d e s}^{e s c_{i}}\right)$ incluso en los escenarios desfavorables.

Tal y como vimos anteriormente, para cada escenario en una fecha $\tau_{j}$ disponemos de su probabilidad de ocurrencia. Además, según lo anterior, asociado a dicho escenario se obtiene un valor de $F_{\text {mod }}^{\tau_{j}}$. Así pues, es posible calcular el valor esperado de esta variable para la distribución de probabilidad existente sobre los escenarios en $\tau_{j}$. Dicho valor lo denotaremos $E\left(F_{\text {mod }}^{\tau_{j}}\right)$ ). Entonces, el criterio de elección de fecha óptima puede enunciarse de la manera siguiente:

$$
\max \left\{E\left(F_{\text {mod }}^{\tau_{1}}\right), E\left(F_{\text {mod }}^{\tau_{2}}\right), \ldots, E\left(F_{\text {mod }}^{\tau_{f}}\right)\right\}
$$

Por otra parte, además de las medidas de riesgo mencionadas anteriormente, el criterio de elección de fecha óptima podría a su vez ser modificado por los gestores del proyecto según otras consideraciones que estimen convenientes, dado el hecho, ya señalado anteriormente, que estamos tratando con un puerto, el cual, además del objetivo de autofinanciación, tiene objetivos de servicio público.

A fin de implementar este método con un ejemplo de discretización anual hemos considerado los siguientes datos:

- Estructura portuaria: es un muelle que soporta 6 tráficos, se consideran como fechas posibles de inversión el año actual y cada uno de los 10 años posteriores (según la notación utilizada, $\tau_{j}=0,1,2, \ldots, 10$; la construcción del muelle requiere 4 años, 
contados a partir de la fecha de inicio de la inversión $\tau_{j}$ considerada y necesitamos conocer el nivel de tráfico de las mercancías (y flujos de caja) en el instante futuro $\tau=\tau_{j}+5$ (inicio de la actividad). Dicho nivel se denotó por $N_{j}(\tau)$.

- Hemos realizado 500 simulaciones para cada $N_{i}(\tau)$, siendo agrupadas mediante un histograma de frecuencias con 7 cortes. De esta forma, los posibles valores futuros de $N_{i}(\tau)$ pueden representarse mediante 7 valores $\left\{N_{i}^{j}\right\}_{i=1}^{7}$, cada uno de los cuales con una determinada probabilidad de ocurrencia. El número total de posibles estados iniciales (escenarios) será $7^{6}=117649$, con su correspondiente probabilidad de ocurrencia. Consideramos sólo las 100000 más probables.

- Se ha establecido un horizonte temporal (vida útil) de 25 años para el cálculo del valor de la función de flujos netos descontados y acumulados $F_{d e s}^{e s c_{i}}$. Hemos realizado cien simulaciones del $F_{d e s}^{e s c_{i}}$, cuya media, $E\left(F_{d e s}^{e s c_{i}}\right)$, es el valor esperado de los flujos netos de caja descontados y acumulados en el instante actual, y se interpreta como la rentabilidad del proyecto si nuestro escenario de partida fuese el considerado. Esto nos permite disponer de 100000 posibles rentabilidades del proyecto, cada una con una probabilidad de ocurrencia asociada (la de su escenario de partida).

- Se ha obtenido finalmente la denominada función $F_{m o d}^{\tau_{i}}$, a partir de las rentabilidades obtenidas, sustituyendo por 0 las de valor negativo. Todas ellas conservan la misma probabilidad asociada. El valor $E\left(F_{\text {mod }}^{\tau_{i}}\right)$ representa el valor del proyecto incorporando la opción de demorar la inversión.

Los valores obtenidos para $E\left(F_{d e s}^{e s c_{i}}\right)$ en los 100000 escenarios considerados, con su correspondiente probabilidad de ocurrencia, cuando la inversión se realiza con 6 años de demora, con 8 años de demora y con 10 años de demora (sin obligación de invertir) muestra que las distribuciones asociadas a 6 y 10 años de demora son menos favorables que la correspondiente a 8 años de demora, ya que, en esta última la cantidad de escenarios favorables (puntos a la derecha del valor 0 en las Figuras 3,4 y 5), así como sus probabilidades asociadas, son ambas más elevadas que en las otras dos. 
Este primer análisis cualitativo se completa con el estudio cuantitativo completo de la secuencia de evolución en el tiempo de $E\left(F_{m o d}^{\tau_{i}}\right)$, valor que mide precisamente el peso específico que los escenarios favorables tienen en el conjunto de la distribución. La Figura 6 nos muestra como el máximo valor de $E\left(F_{m o d}^{\tau_{i}}\right)$ se alcanza cuando la inversión se realiza con 8 años de demora con respecto a la fecha actual.

Por último, ilustramos en la Figura 7 una de las medidas de riesgo complementarias al criterio de decisión descrito, consistente en determinar para cada distribución, la probabilidad de ocurrencia de escenarios desfavorables. De este modo, no sólo disponemos de $E\left(F_{m o d}^{\tau_{i}}\right)$ (que será siempre un valor positivo), sino del riesgo inherente a admitir dicho valor como estimador del valor proyecto. Observamos como existe una correlación clara entre valores crecientes del proyecto y menor probabilidad de aparición de escenarios desfavorables, con un punto de inflexión en el mismo año 8.

\section{3}

La combinación de fechas de construcción para un porcentaje dado de inversión públicaprivada genérico, se recoge en la Tabla 1, donde denotamos por las letras O y S los dos muelles cuya construcción es opcional, y se añade un dígito para indicar los años de demora de la inversión respecto de la fecha de partida. En este ejemplo consideraremos, por una parte, como fecha de partida el 2007 y 5 años posibles de demora en ambos muelles. Por otra parte, suponemos que el muelle $\mathrm{S}$ es el que admite un posible porcentaje de inversión privada (la elección responde al hecho de que posee menos tráficos y disminuye el coste computacional del problema).

De este modo, el problema de decisión de fecha óptima para los muelles $O$ y $S$, y de elección del porcentaje de inversión pública-privada en el muelle Nuevo Sur, se puede formular de la forma siguiente: Para cada nivel de porcentaje, se debe encontrar la combinación de fechas de inversión para cada muelle de modo que se maximice la suma de valores esperados de las funciones de flujos modificadas (Tabla 1). Comparando los máximos alcanzados en cada nivel, se elige el porcentaje óptimo como aquel para el que su combinación de fechas óptimas asociada tiene la mayor suma de valores esperados.

Este criterio de maximización puede ampliarse introduciendo medidas cuantitativas de riesgo, como por ejemplo, alguna condición sobre la probabilidad de que en un instante del tiempo los valores esperados de flujos netos descontados asociados a los escenarios sean 


\begin{tabular}{c|cccccc|c}
\hline \hline & \multicolumn{7}{|c}{ Muelle Oeste } \\
Muelle Sur & 2007 & 2008 & 2009 & 2010 & 2011 & 2012 & \\
\hline 2007 & $\mathrm{O}+\mathrm{S}$ & $\mathrm{O} 1+\mathrm{S}$ & $\mathrm{O} 2+\mathrm{S}$ & $\mathrm{O} 3+\mathrm{S}$ & $\mathrm{O} 4+\mathrm{S}$ & $\mathrm{O} 5+\mathrm{S}$ & $\mathrm{S}$ \\
2008 & $\mathrm{O}+\mathrm{S} 1$ & $\mathrm{O} 1+\mathrm{S} 1$ & $\mathrm{O} 2+\mathrm{S} 1$ & $\mathrm{O} 3+\mathrm{S} 1$ & $\mathrm{O} 4+\mathrm{S} 1$ & $\mathrm{O} 5+\mathrm{S} 1$ & $\mathrm{~S} 1$ \\
2009 & $\mathrm{O}+\mathrm{S} 2$ & $\mathrm{O} 1+\mathrm{S} 2$ & $\mathrm{O} 2+\mathrm{S} 2$ & $\mathrm{O} 3+\mathrm{S} 2$ & $\mathrm{O} 4+\mathrm{S} 2$ & $\mathrm{O} 5+\mathrm{S} 2$ & $\mathrm{~S} 2$ \\
2010 & $\mathrm{O}+\mathrm{S} 3$ & $\mathrm{O} 1+\mathrm{S} 3$ & $\mathrm{O} 2+\mathrm{S} 3$ & $\mathrm{O} 3+\mathrm{S} 3$ & $\mathrm{O} 4+\mathrm{S} 3$ & $\mathrm{O} 5+\mathrm{S} 3$ & $\mathrm{~S} 3$ \\
2011 & $\mathrm{O}+\mathrm{S} 4$ & $\mathrm{O} 1+\mathrm{S} 4$ & $\mathrm{O} 2+\mathrm{S} 4$ & $\mathrm{O} 3+\mathrm{S} 4$ & $\mathrm{O} 4+\mathrm{S} 4$ & $\mathrm{O} 5+\mathrm{S} 4$ & $\mathrm{~S} 4$ \\
2012 & $\mathrm{O}+\mathrm{S} 5$ & $\mathrm{O} 1+\mathrm{S} 5$ & $\mathrm{O} 2+\mathrm{S} 5$ & $\mathrm{O} 3+\mathrm{S} 5$ & $\mathrm{O} 4+\mathrm{S} 5$ & $\mathrm{O} 5+\mathrm{S} 5$ & $\mathrm{~S} 5$ \\
& $\mathrm{O}$ & $\mathrm{O} 1$ & $\mathrm{O} 2$ & $\mathrm{O} 3$ & $\mathrm{O} 4$ & $\mathrm{O} 5$ & - \\
\hline \hline
\end{tabular}

Tabla 1:

negativos, los escenarios adversos.

De este modo, para cada casilla de la Tabla 1 se obtiene una pareja de medidas de riesgo. Combinando los resultados obtenidos como suma de los valores esperados de las funciones de flujos modificadas, y las probabilidades correspondientes, es posible utilizar procedimientos clásicos de selección de proyectos según rentabilidad-riesgo para la obtención de una fecha de inversión, la cual no tiene porqué coincidir con la que se obtendría al utilizar únicamente un criterio de valor esperado.

\section{Método de resolución del problema discreto}

Debemos observar que la técnica de búsqueda del óptimo basada en la exploración exhaustiva no es generalizable a problemas en los que exista un mayor número de variables de decisión y/o éstas tengan un rango de variación más amplio. En dichos problemas, el coste computacional de evaluar la función de flujos netos descontados para todas las combinaciones posibles de las variables de decisión es tan elevado que es impracticable, por lo que se hace necesaria una técnica de búsqueda más sofisticada que explore selectivamente el espacio de combinaciones de los diversos valores.

Este modelo está sujeto al nivel de discretización que los gestores fijen para las dos variables (fechas de inicio y porcentajes de participación pública) y, si así se decide la variación de los tipos de interés.

Así pues, dada una parrilla de puntos caracterizados cada uno de ellos por los tres valores, se trata de encontrar un punto que proporcione una buena solución que vendrá determinada por el mayor Valor esperado del proyecto posible dentro de un nivel aceptable de probabilidad de abandono.

Esta tipo de problema encaja, en buena parte, con la metodología de los procedimientos aproximados de optimización de última generación conocidos como metaheurísticos. Existen de diversas clases y orígenes y los más consolidados en la resolución de problemas reales son los conocidos como Búsqueda Tabú (Tabu Search), el Templado Simulado (Simulated Annealing) y los Algoritmos Genéticos (GA). En los últimos años han aparecido otros métodos con resultados muy prometedores entre los que destacan el método Grasp (Greedy Randomized Adpative Search Procedure) y la Búsqueda Dispersa (Scatter Search). 
El objetivo de todas estas técnicas es realizar la exploración de un espacio de valores sobre el que se tiene un conocimiento escaso de manera que se obtenga el mejor de los valores, o en su defecto uno razonablemente bueno, consiguiéndose en un tiempo de computación aceptable para el usuario. Aunque el objetivo señalado de los metaheurísticos encaja perfectamente con el que tenemos planteado en nuestro problema existe una diferencia importante que consiste en el hecho de que todos los métodos metaheurísticos suelen requerir la realización de gran cantidad de evaluaciones de puntos para poder determinar los criterios de búsqueda. Desde el momento en que se comprobó el tiempo necesario para cada simulación quedó claro que la dificultad principal del problema estribaba en el tiempo de las simulaciones. Por todo ello hemos optado plantear un procedimiento tan ajustado como nos ha sido posible basado en las ideas de la Búsqueda Dispersa (SS) [GLM01].

Hemos tratado de establecer una serie de reglas de mejora en el movimiento de un punto a otro aplicables a cualquier versión del problema. Los criterios de movimiento deben tener en cuenta la estructura de la función a optimizar y explotar el conocimiento previo de la función objetivo ante variaciones de un único control mientras los demás permanecen constantes. En nuestro caso, la función objetivo es una compleja simulación por lo que, aunque se intuían algunas tendencias, no podemos establecer reglas de manera general.

\subsection{El algoritmo planteado}

Seguimos las ideas básicas del Scatter Search conocedores de las limitaciones para calcular el valor de la función objetivo en muchos puntos, hecho que nos obliga a ser muy selectivos al evaluar. Algunos de los criterios establecidos en la metodología de búsqueda, se concretan en el caso particular de nuestro problema en los siguientes puntos:

- Las combinaciones entre puntos no produce uno de la parrilla se establece un redondeo aleatorio.

- Si una combinación no convexa produce un punto externo a la parrilla escogeremos el punto de la parrilla más próximo a él.

- Dado el elevado coste de evaluar cada uno de los puntos, se crea una memoria histórica de puntos visitados con su evaluación correspondiente. Esto supone muy poco coste de memoria, dado que la exploración es muy limitada, y permite un gran ahorro si se ha de evaluar un punto ya evaluado.

- El criterio de parada está determinado por una cantidad fija de evaluaciones. Como el tiempo de cada evaluación está bastante bien estimado fijamos aproximadamente la duración del proceso.

- Los gestores pueden fijar un nivel mínimo de probabilidad de realización de la inversión por debajo del cual un punto no sería aceptable. Sin embargo, con la finalidad de no desconectar el espacio de soluciones estos puntos no aceptables, aunque no serán nunca una solución a ofrecer, serán válidos como soluciones de tránsito ya que en su entorno puede haber puntos aceptables. 
- Hemos fijado una cantidad mínima de 80 evaluaciones ya que por debajo de esa cantidad el proceso es muy corto. La hemos respetado incluso para el ejemplo más pequeño con una parrilla de unos 200 puntos. La cantidad de evaluaciones debe incrementarse con el aumento del número de puntos pero no linealmente y no debería rebasar una cota máxima según el tiempo que los decisores consideren aceptable.

Exponemos a continuación el algoritmo de Búsqueda Dispersa adaptado a nuestro contexto. Esta adaptación lleva a una reducción drástica de los puntos considerados para limitar al máximo el número de evaluaciones realizadas:

Paso 1.- Escoger una serie de puntos dispersos y evaluarlos. El criterio seguido es coger y combinar 3 valores de cada parámetro (los extremos y el intermedio). Estos 27 puntos constituyen el conjunto inicial.

Paso 2.- Formar el Conjunto de Referencia (RS). El conjunto RS consta de 6 puntos $(R S=S C \cup S D)$. Los 3 mejores (respecto al VAN) encontrados formarán el Subconjunto de Calidad (SC) inicial del RS. Además formaremos el Subconjunto de Diversificación (SD) con 3 puntos que serán los más lejanos del Subconjunto de Calidad. La distancia utilizada viene dada por la expresión

$$
\max _{x \in I}\left\{\min _{y \in S C} d(x, y)\right\}
$$

(calculada la distancia de cada punto de I al más próximo de SC, elegimos los que tengan las 3 mayores distancias).

Paso 3.- Fase de Búsqueda Local (LS). Desde los puntos que forman el subconjunto de calidad del RS realizamos un proceso de Búsqueda Local. Este proceso se basa en la Primera Mejora (First Improving), es decir, en cuanto encontramos un punto mejor que el actual nos desplazamos a él sin necesidad de explorar todo su entorno. Dado que la ubicación de los puntos es homogénea en el espacio (con excepción de las fronteras del mismo) hemos fijado también un criterio arbitrario de exploración.

Paso 4.- Combinar los elementos del RS y actualizar RS.

4.1. Combinar los elementos del RS. Realizaremos todas las combinaciones posibles (15) entre los elementos del Conjunto de Referencia. stas serán tanto convexas como no convexas. La combinación convexa elige el punto medio entre ellos. Los no convexos serán los puntos de la recta externos al segmento que se encuentran a una distancia igual a la mitad de la que separa a ambos. Es decir, elegidos los puntos $x_{1}$ y $x_{2}$ a combinar, consideraremos los puntos de $x_{1} d \mathrm{y}, x_{2}+d$ donde $d=\left(x_{2}-x_{1}\right) / 2^{1}$.

4.2. Actualización de RS. De esta forma obtenemos 45 puntos, 3 para cada combinación. De entre ellos y los que estaban en el RS elegiremos los 6 mejores para formar el nuevo RS.

4.3. Si se han agotado las iteraciones, paramos.

Paso 5.- Nueva Búsqueda Local (LS). Desde los 3 mejores puntos de RS realizamos

\footnotetext{
${ }^{1}$ Existe la opción de hacer estas combinaciones aleatorias aunque, no es el procedimiento que estamos implementando en primera instancia. En ese caso, se elegiría un número aleatorio $r$ en el intervalo $[0,1] l$ y la combinación convexa sería $r\left(x_{2}-x_{1}\right)+x_{1}$. Los puntos resultado de las combinaciones no convexas serían $x_{1} d$ y $x_{2}+d$ donde $d=r\left(x_{2}-x_{1}\right) / 2$.
} 
un nuevo proceso de Búsqueda Local con los criterios ya señalados. Si hemos agotado las iteraciones, paramos.

Paso 6.-

- Si no se ha modificado RS, diversificarlo eliminando las 3 peores soluciones y añadiendo 3 nuevas soluciones diversas como se hizo en el paso 2.

- Volver al paso 4.

Como se observa el algoritmo intenta combinar un criterio inicial de calidad con otro de diversidad para, a continuación, guiarse sólo por criterios de calidad en un proceso convergente. Si el número de iteraciones fuese suficientemente grande se produciría una situación de estancamiento que se reactiva mediante la reconstrucción del conjunto de referencia que introduce diversidad prevista en el paso 6. No obstante, en nuestra situación o bien el tiempo disponible es muy grande o esto no llegará a suceder porque el proceso de exploración es muy corto desde el criterio de puntos visitados.

\subsection{Pruebas experimentales}

El algoritmo desarrollado hasta el momento se ha implementado en $\mathrm{C}++\mathrm{y}$ trabaja en un procesador AMD a $650 \mathrm{Mhz}$. El tiempo que emplea en la realización de los movimientos es totalmente despreciable comparado con el coste de las evaluaciones (30 minutos para hacer la simulación y evaluar cada punto). El ejemplo expuesto al final del tercer apartado está discretizado por años del 2007 al 2012 para la construcción de ambos muelles y porcentajes de capital público en el muelle Oeste del 25\%, 50\%, 75\% y $100 \%$.

La suma de valores esperados de las funciones de flujos modificadas para cada combinación de fechas de inicio de la actividad de los muelles Oeste y Nuevo Sur, cuando el porcentaje de inversión pública en el muelle Sur se ha fijado en el 100\%, presenta el óptimo cuando la fecha de inicio de la actividad del muelle Oeste es el 2011, y del muelle Nuevo Sur, el 2007 y la probabilidad de no-realización de la inversión es del 54.76\% (obtenida como máximo de las probabilidades de no inversión de los dos muelles separadamente).

Si repetimos el proceso cuando el porcentaje de inversión pública es del $75 \%$, se obtiene el óptimo para la misma combinación de fechas pero con una suma de valores esperados de flujos inferior a la que se obtenía con un $100 \%$ de inversión pública. Conservamos el primer resultado y continuamos la búsqueda.

Con un $50 \%$ de inversión pública se reproduce la situación planteada para el caso del $75 \%$.

En la Figura 8 representamos los valores obtenidos para el nivel del 25\%. La combinación de fechas óptimas se mantiene en el 2011 para el muelle Oeste y el 2007 para el muelle Nuevo Sur. Sin embargo, ahora la suma de valores esperados sí que es superior a la del nivel del 100\%. Así pues, podemos asegurar que el óptimo de todo el espacio de decisión se alcanza para las fechas indicadas y una inversión pública del $25 \%$.

En la Figura 9 se representan las probabilidades de no-realización de la inversión para cada combinación de fechas, para el porcentaje público del $25 \%$, nivel en el que se alcanza el óptimo global. Observamos que la probabilidad asociada a dicho óptimo es del 41,63\% (inferior al 54,76\%, nivel del 100\%). 
En un análisis más detallado de la superficie completa se observa que la combinación de fechas dada por la no construcción del muelle Oeste y el inicio en el 2007 de la actividad del muelle Nuevo Sur, tiene una probabilidad de no-realización únicamente del 3\% y una suma de valores esperados sólo ligeramente inferior a la del óptimo global. Este hecho podría ser tomado en consideración por los gestores del proyecto para decidir su ejecución final. Así pues, la combinación de las Figuras 8 y 9 permitirá diseñar criterios mixtos rentabilidad-riesgo de búsqueda de la combinación óptima de las variables de decisión del problema.

Otras pruebas se han hecho con parrillas más densas (porcentajes a saltos del $10 \%$ y discretización por trimestres) y los resultados encontrados no difieren más del $10 \%$ de la mejor solución conocida por lo que el sistema de exploración resulta prometedor. Sin embargo, es necesario aumentar la discretización y continuar las pruebas para poder asegurar que el método funciona suficientemente bien para proponerlo en general.

Además, es necesario probar con diversos valores para la tasa de interés, puesto que ésta influye considerablemente en la simulación.

Otras pruebas se han hecho con parrillas más densas (porcentajes a saltos del $10 \%$ y discretización por trimestres) y los resultados encontrados no difieren más del $10 \%$ de la mejor solución conocida por lo que el sistema de exploración resulta prometedor. Sin embargo, es necesario aumentar la discretización y continuar las pruebas para poder asegurar que el método funciona suficientemente bien para proponerlo en general.

Además, es necesario probar con diversos valores para la tasa de interés, puesto que ésta influye considerablemente en la simulación.

Figura 8

Figura 9

\section{Referencias}

[AP99] Autoridad Portuaria de Valencia (1999) Annual Report. 
[BS73] Black, F.; Scholes, M. (1973) "The pricing of options and corporate liabilities", Journal of Political Economy 81: 637-659.

[CA01] Copeland, T.; Antikarov, V. (2001) Real Options, A practitioner's Guide. TEXERE LLC.

[COPR00] Casasús, T.; Olmos, F.; Pérez, J.C.; Rodrigo, A. (2000) "Valoración de utilidades públicas: un enfoque de opciones reales", VIII Foro de Finanzas, Madrid.

[DP94] Dixit; Pindyck (1994) Investment under Uncertainty. Princeton University Press.

[GL97] Glover, F.; Laguna, M. (1997) Tabu Search. Kluwer Academic Publishers

[GLM01] Glover, F.; Laguna, M.; Martí, R. (2001) "Scatter search", to appear in Theory and Applications of Evolutionary Computation: Recent Trends, in: Ghosh \& Tsutsui (Eds.), Springer-Verlag.

[M73] Merton, R.C. (1973) "Theory of rational option princing", Bell Journal of Economics and Management Science 4: 141-183.

[R95] Ross, S. (1995) "Uses, abuses, and alternatives to the net-present-value rule", Financial Management 24(3): 96-102.

[T96] Trigeorgis, L. (1996) "Real Options in Capital Investment: Models, Strategies and Applications". Praeger, Westport CT.

[W00] Wilmott, P. (2000) Quantitative Finance. John Wiley \& Sons, New York. 